\title{
EFFECT OF GENOTYPE AND SOME ENVIRONMENTAL FACTORS ON MILK YIELD, MILK COMPOSITION, SOMATIC CELL COUNTS AND BACTERIAL COUNT OF LOCAL BARKI, DAMASCUS GOATS AND THEIR CROSSES
}

\author{
M.M. Eissa and Mona A. Osman
}

Animal Production Research Institute, Ministry of Agriculture and Land Reclamation, Dokki, Giza, Egypt

\section{SUMMARY}

Desert Barki (B), Damascus (D) goats, $F_{1}$ (crosses D.B) and the backcrosses $\{D$ $X(D . B)\}\{B X(D . B)\}$ were studied under semi-arid conditions of the coastal zone of the western desert of Egypt in 2002. Monthly milk samples were taken from studied genotypes at morning and evening milking times to estimate somatic cell counts (SCC), daily milk yield (DMY), percentages of fat, protein, lactose and total solids and bacterial counts. Also, partial correlation coefficient was estimated between pairs of all studied traits. Least squares means indicated that Damascus produced higher daily milk yield than Barki goats in the morning $(0.330 \mathrm{~kg}$ vs $0.290 \mathrm{~kg})$. Among crossbred does, backcrosses had higher DMY than D.B. The effect of genotype was significant on protein and lactose percentages (morning milking) and $D M Y$ (evening milking). There was no significant effect of parity on the studied traits except for DMY in the morning milking. Type of kidding did not affect the studied traits except lactose percentage from morning milk samples. Order of lactation month had significant effects on SCC, DMY and all milk constituents except lactose percentage in the evening milking. Contagious pathogens were affected by genotypes except Staph. aureus. Parity had significant effect on Corinebacteria and Strep. agalactiae in the morning milking and Strep. dysgalactiae in the evening milking. The influence of type of kidding was significant on contagious pathogens except Staph. aureus in the morning milking. Order of lactation months affected Strep. dysgalactiae in both milkings. Bacillus as an environmental pathogen was affected by genotype for both milkings, while type of kidding affected Strep. uberis. Order of lactation months did not show significant effect on environmental pathogens. SCC had a positive relationship $(p<0.01)$ with fat $\%$, protein $\%$, total solids $\%$ and contagious pathogens, while it had a negative relationship $(p<0.01)$ with DMY $(\mathrm{kg})$ and lactose \% in both morning and evening milkings. DMY negatively correlated $(p<0.01)$ with fat \%, protein \%. SCC is considered one of the most important methods to evaluate the health condition of goats udder.

Keywords: Somatic cell counts, milk composition, bacteriological examination, partial correlation coefficients

\section{INTRODUCTION}

Milk somatic cell counts (SCC) is widely used to monitor udder health and, thus, milk quality. SCC from a day's milk is the best indicator of the extent to which the

Issued by The Egyptian Society of Animal Production 
gland is involved in fighting a mastitis infection. When udder tissue is injured or becomes infected, significant numbers of white blood cells accumulate in the milk and milk yield is depressed and composition may be altered. Normal goat milk has a higher cell count than normal milk from cows. Dairy goats have a different secretory system from cows, due to its nature of milk secretion. The apocrine system of goats produces cytoplasmic particles and their milk may contain a large number of epithelial cells (Park and Humphrey, 1986). The presence of mastitis infection in dairy goat herds is reflected in bulk milk tank samples with a somatic cell count exceeding 1,000,000 cells per milliliter. The current Pasteurized Milk Ordinance (PMO) regulation (PMO, 1993) regarding somatic cell count (SCC) in goat milk allows $1 \times 10^{6} \mathrm{SCC} / \mathrm{ml}$ milk. With the support of pathological and histological evidence in udder tissues, Zeng and Escobar (1995) concluded that SCC of more than 1 million in milk did not indicate any mastitis condition in Alpine goats, agreeing with previous finding of Maisi, (1990), Haenlein and Hinckley (1995) and El-Saied et al. (2003) for different goat breeds. Bacteria usually, but not always, can be isolated in milk. High SCC are associated with bacterial intramammary infection, but other factors such stage of lactation, parity, and infection by caprine arthritisencephalitis virus, also contribute to elevated SCC (Dulin et al. 1983). Also, Poutrel et al. (1997) indicated that numerous factors such as farm, breed, age, stage of lactation, estrus, milk production, management conditions, and intramammary infections have been mentioned to affect somatic cell counts in goat milk.

The objectives of this research were to: (a) study the influence of genotype of doe, parity of doe, litter size of milking doe and the order of month of lactation on somatic cell count (SCC), daily milk yield, milk constituents and microbiological assay SPC; (b) estimate correlations between studied variables.

\section{MATERIALS AND METHODS}

\section{Data and herd management}

The present study was carried out at Borg EL-Arab experimental station belonging to the Animal Production Research Institute (APRI), Egyptian Ministry of Agriculture and Land Reclamation, over the complete lactation (February-September, 2002) on 964 milk samples from 101 does $\{37$ Damascus (D), 9 Barki (B), 26 $\left(3 / 4 \mathrm{D}^{1 / 4 \mathrm{~B}}\right), 15\left(1 / 2 \mathrm{D}^{1 / 2} \mathrm{~B}\right)$ and $14\left(1 / 4 \mathrm{D}^{3} / 4 \mathrm{~B}\right)$. Animals were kept in confinement all year round and fed Egyptian clover hay (Trifolium alexandrinum) and barley straw in addition to concentrate supplement according to their needs. Animals were allowed to drink twice daily. Mating season started in July and lasted for 90 days. Kidding took place during December and January. Kids were kept with their dams up to weaning at 90 days.

\section{Milk sample collection}

Records of daily milk yield, milk composition, SCC and bacterial cell count were taken at monthly intervals following an a.m./p.m. recording scheme. Milk samples were taken from each half udder (almost $15 \mathrm{ml}$ ) in sterilized tubes and kept in an icebox at $4{ }^{\circ} \mathrm{C}$ until bacteriological examination. Preserved samples were delivered to the laboratory within 3 hours after sampling. 


\section{Analysis of milk composition, SCC determination and Bacterial examination}

Milk composition traits (fat $\%$, protein $\%$, lactose $\%$ and total solids \%) were measured by the automated method of infrared absorption spectrophotometry (Milko-Scan; Foss Electric, Hillerd, Denmark) and SCC were determined using the Fossmoatic method with Fossmoatic machine and the machine was calibrated for goat milk. Total bacteria counts were investigated through standard plate count (SPC) according to the microbiological count method of Houghtby et al. (1992). A portion of each aseptic sterile vial sample was diluted 10 times with quarter strength Ringer's solution, $1 \mathrm{ml}$ of this sample was spread on a sheep blood agar plate (SBA), incubated aerobically at $37^{\circ} \mathrm{C}$ and examined at 24 and $48 \mathrm{~h}$ for counting the number of colonies. Individual colonies were sub cultured Gram's staining, Catalase test and Coagulase test were performed to purify and classify the Coliform, Streptococci, Coagulase-Negative Staphylococci (CNS) and Staphylococcus aureus species according to the method of Higgs and Bramely (1981). Milk composition analysis, SCC determination and bacteriological investigation were carried out at the Dairy Services Unit of Animal Production Research Institute ( Sakha, Kafr El- Shaiekh ).

\section{Statistical analysis}

Data were analyzed separately for morning and evening milk samples using the same model. Least-squares for SCC, DMY, percentages of fat, protein, lactose and total solids and microbiological assay (SPC) were applied, using the Mixed Models procedure of the SAS Institute Inc. (1996). This procedure was used to analyze the repeated measurements.

The following model was applied for morning and evening milking separately to obtain estimates for the investigated traits: Where:

$$
Y_{i j k l m n}=\mu+b_{i}+p_{j}+s_{j}+a(b p s)_{i j k l}+o_{m}+e_{i j k l m n}
$$

$\mathrm{Y}_{\mathrm{ijklmn}}=$ records of somatic cell count (SCC), daily milk yield (DMY), fat \%, protein $\%$, lactose $\%$, total solids $\%$ and SPC,

$\mu=$ the overall mean,

$b_{i}=$ the fixed effect of genotypes, $i=1,2,3,4,5$ for Barki , Damascus, $\left(1 / 2 D^{1 / 2} B\right)$, $\left(3 / 4 \mathrm{D}^{1 / 4} \mathrm{~B}\right)$, and $\left(1 / 4 \mathrm{D}^{3 / 4} \mathrm{~B}\right)$, respectively,

$\mathrm{p}_{\mathrm{j}}=$ the fixed effect of parity,

$j=1,2, \ldots \ldots, \geq 6, s_{k}=$ the fixed effect of litter size of the milking doe,

$\mathrm{k}=1,2, \mathrm{a}(\mathrm{bps})_{\mathrm{ijk} \mathrm{l}}=$ the random effect of animal $\mathrm{i}$, nested within genotype $\mathrm{i}$, parity $\mathrm{j}$ and litter size $\mathrm{k}$,

$\mathrm{o}_{\mathrm{m}}=$ the fixed effect of months order throughout the lactation period,

$\mathrm{m}=1.2 \ldots . .7$, and $\mathrm{e}_{\mathrm{ijklmn}}=$ random error.

SCC and SPC data were measured as 100,000 (hundred thousand) cells per ml then transformed to their logarithmic form (log base 10) to meet the statistical requirements for hypothesis testing (Ali and Shook, 1980).

Partial correlation coefficients were estimated using the same models for morning and evening milk samples from the Error SS and CP Matrix / Prob $>\mid \mathrm{r}$ between each pair of $\log 10 \mathrm{SCC}, \mathrm{DMY}$, the percentages of fat, protein, lactose and total solids and SPC, using the GLM procedure of the SAS Institute Inc. (1996). Also this procedure handles the repeated measurements. 


\section{RESULTS AND DISCUSSION}

\section{The effect of environmental factors on SCC, DMY and milk composition}

Least squares means \pm standard errors for SCC, DMY and milk composition in different levels of fixed effects during a complete lactation from morning and evening milk samples are given in tables 1 and 2, respectively. Overall mean of SCC of all genotypes in the present study was estimated as $5.24 \times 10^{5}$. The discriminating threshold values between healthy and infected udders found in the literature ranged from $8.0 \times 10^{5}$ to $2 \times 10^{6} \mathrm{SCC} / \mathrm{ml}$ milk (Dulin et al., 1983, PMO, 1993, Hinckley, 1993, Wilson et al., 1995, Zeng and Escobar, 1996, Haenlein, 2000 and El-Saied et al., 2003).

Tables 1 and 2 show that no significant differences were observed for $\log 10$ SCC for studied genotypes. The present results are in agreement with Osman et al. (2004) who showed that no significant differences for SCC were detected between Damascus and Baladai goats. Also, Zeng and Escobar (1996) reported no significant difference between Alpine and Nubian for SCC. In contrast to this study, Sheldrake et al. (1981) reported a significant variation in SCC among goat herds, ranging from $4.38 \times 10^{5}$ to $1.68 \times 10^{6} \mathrm{SCC} / \mathrm{ml}$ milk. Park and Humphrey (1986) analyzed milk samples from mid-lactation of Alpine and Nubian does and found a higher SCC in Nubian milk than it in Alpine milk. Sung et al. (1999) found significant variation in SCC between Alpine, Nubian, Saanen and Toggenburg in Taiwan. The genotype had significant effect on protein and lactose and non significant on DMY, fat and total solids in the morning milking, while it had significant effect on DMY and a non significant effect on all milk constituents in the evening milking. Damascus does produced higher daily milk yield than that of all other genotypes in the morning milking.

The effects of parity of doe and type of kidding were not significant on SCC and milk composition (tables 1 and 2), however, DMY at morning time was affected by parity of doe. DMY had the highest estimate in the third parity which might have been due to the udder development with the progress of the age of doe and also the development of digestive system, therefore dry matter intake and as consequence milk yield is increased. Similar results were reported by Prasad and Sengar (2002) and Abo-Ismail (2003). DMY was higher for goats that gave twins than for single kidding goats. Hatfield et al. (1995) suggested that this effect may be attributed to physiological mechanisms during pregnancy that prepare the udder to produce more milk when a doe is carrying multiple fetuses. The present results are in agreement with those of Hayden et al. (1979), Hatfield et al. (1995), Prasad and Sengar (2002) and Abo-Ismail (2003).

Order of lactation months affected all considered traits in tables 1 and 2. SCC decreased from the second to sixth month of lactation in the evening milking, while it did not show a particular pattern in the morning milking. Wilson et al. (1993) indicated that increasing days in milk and month of year were among the most important factors contributing to increased cell count in the absence of intramammary infection. DMY declined gradually after the first two month freshening, while the milk protein increased gradually with the advance of the stage of lactation in the morning milking. No particular trend was observed for other milk composition either 
in morning or evening milkings. These results were in good agreement with the findings of Prasad and Sengar (2002) and Osman et al. (2004).

Table 1. Morning milk samples, Least squares means, standard error $( \pm \mathrm{SE})$ and probability of type I error (P) for $\log 10$ (SCC), DMY, percentages of fat, protein, lactose, total solids

\begin{tabular}{|c|c|c|c|c|c|c|c|}
\hline \multirow[t]{2}{*}{ Factor } & \multirow[t]{2}{*}{ No } & $\begin{array}{l}\log 10 \\
(\mathrm{SCC})\end{array}$ & DMY, kg & Fat $\%$ & Protein \% & $\begin{array}{l}\text { Lactose } \\
\%\end{array}$ & $\begin{array}{l}\text { Total } \\
\text { solid \% }\end{array}$ \\
\hline & & $\begin{array}{c}\text { Mean } \pm \text { SE } \\
\text { P }\end{array}$ & $\begin{array}{c}\text { Mean } \pm \text { SE } \\
\mathbf{P}\end{array}$ & $\begin{array}{l}\text { Mean } \pm \text { SE } \\
\mathbf{P}\end{array}$ & $\begin{array}{l}\text { Mean } \pm \text { SE } \\
\text { P }\end{array}$ & $\begin{array}{l}\text { Mean } \pm \mathbf{E} \\
\mathbf{P}\end{array}$ & $\begin{array}{l}\text { Mean } \pm \text { SE } \\
\mathbf{P}\end{array}$ \\
\hline Genotype: & & 0.06 & 0.09 & 0.26 & 0.04 & 0.04 & 0.95 \\
\hline Barki(B) & 9 & $5.45 \pm .08$ & $0.290 \pm .02$ & $3.83 \pm .11$ & $3.55 \pm .08$ & $4.09 \pm .06$ & $11.64 \pm .16$ \\
\hline Damascus (D) & 37 & $5.33 \pm .06$ & $0.330 \pm .01$ & $3.56 \pm .08$ & $3.45 \pm .06$ & $4.27 \pm .04$ & $11.64 \pm .11$ \\
\hline $3 / 4 \mathrm{D} * 1 / 4 \mathrm{~B}$ & 26 & $5.20 \pm .07$ & $0.304 \pm .02$ & $3.55 \pm .09$ & $3.39 \pm .07$ & $4.21 \pm .05$ & $11.72 \pm .12$ \\
\hline $1 / 2 \mathrm{D} * 1 / 2 \mathrm{~B}$ & 15 & $5.22 \pm .09$ & $0.266 \pm .02$ & $3.64 \pm .12$ & $3.66 \pm .08$ & $4.11 \pm .06$ & $11.70 \pm .16$ \\
\hline $3 / 4 \mathrm{~B} * 1 / 4 \mathrm{D}$ & 14 & $5.44 \pm .09$ & $0.308 \pm .02$ & $3.71 \pm .12$ & $3.44 \pm .08$ & $4.12 \pm .06$ & $11.58 \pm .16$ \\
\hline Parity: & & 0.45 & 0.01 & 0.54 & 0.45 & 0.80 & 0.54 \\
\hline 1 & 26 & $5.33 \pm .07$ & $0.298 \pm .02$ & $3.55 \pm .10$ & $3.54 \pm .07$ & $4.15 \pm .05$ & $11.60 \pm .13$ \\
\hline 2 & 22 & $5.22 \pm .07$ & $0.282 \pm .02$ & $3.63 \pm .09$ & $3.58 \pm .07$ & $4.10 \pm .05$ & $11.55 \pm .13$ \\
\hline 3 & 27 & $5.29 \pm .06$ & $0.344 \pm .01$ & $3.74 \pm .08$ & $3.46 \pm .06$ & $4.15 \pm .04$ & $11.74 \pm .11$ \\
\hline 4 & 10 & $5.27 \pm .09$ & $0.310 \pm .02$ & $3.62 \pm .11$ & $3.54 \pm .08$ & $4.14 \pm .06$ & $11.46 \pm .16$ \\
\hline 5 & 4 & $5.47 \pm .14$ & $0.308 \pm .03$ & $3.68 \pm .20$ & $3.44 \pm .13$ & $4.24 \pm .11$ & $11.88 \pm .27$ \\
\hline$\geq 6$ & 12 & $5.41 \pm .08$ & $0.255 \pm .02$ & $3.75 \pm .12$ & $3.43 \pm .08$ & $4.20 \pm .06$ & $11.70 \pm .16$ \\
\hline $\begin{array}{l}\text { Type of } \\
\text { kidding: }\end{array}$ & & 0.14 & 0.25 & 0.49 & 0.16 & 0.00 & 0.91 \\
\hline 1 & 69 & $5.38 \pm .05$ & $0.291 \pm .01$ & $3.63 \pm .06$ & $3.46 \pm .05$ & $4.24 \pm .03$ & $11.65 \pm .08$ \\
\hline 2 & 32 & $5.28 \pm .05$ & $0.308 \pm .01$ & $3.69 \pm .08$ & $3.54 \pm .06$ & $4.09 \pm .04$ & $11.66 \pm .10$ \\
\hline $\begin{array}{l}\text { Month of } \\
\text { lactation }\end{array}$ & & 0.00 & 0.00 & 0.00 & 0.00 & 0.00 & 0.00 \\
\hline 1 & 101 & $5.39 \pm .06$ & $0.407 \pm .02$ & $3.55 \pm .09$ & $3.16 \pm .05$ & $4.25 \pm .05$ & $11.51 \pm .11$ \\
\hline 2 & 87 & $5.43 \pm .07$ & $0.357 \pm .02$ & $3.51 \pm .11$ & $3.26 \pm .07$ & $4.27 \pm .06$ & $11.48 \pm .19$ \\
\hline 3 & 76 & $5.09 \pm .09$ & $0.294 \pm .02$ & $3.32 \pm .09$ & $3.35 \pm .07$ & $4.10 \pm .07$ & $11.15 \pm .18$ \\
\hline 4 & 69 & $4.83 \pm .11$ & $0.283 \pm .02$ & $3.80 \pm .10$ & $3.36 \pm .08$ & $4.10 \pm .06$ & $11.78 \pm .14$ \\
\hline 5 & 64 & $5.34 \pm .09$ & $0.262+ \pm .02$ & $3.74 \pm .11$ & $3.69 \pm .13$ & $3.93 \pm .06$ & $11.56 \pm .14$ \\
\hline 6 & 51 & $5.53 \pm .08$ & $0.258 \pm .02$ & $3.95 \pm .14$ & $3.93 \pm .15$ & $4.10 \pm .07$ & $12.15 \pm .15$ \\
\hline 7 & 34 & $5.71 \pm .07$ & $0.236 \pm .02$ & $3.74 \pm .16$ & $3.73 \pm .22$ & $4.38 \pm .08$ & $11.96 \pm .24$ \\
\hline
\end{tabular}

\section{The effect of environmental factors on bacteria cell count}

Maisi (1990) and Droke et al. (1993) reported that diagnoses of goat mastitis depend on clinical symptoms but non-clinical mastitis can be diagnosed better when organisms from the milk are identified than SCC. Least squares means and \pm standard errors of Standard Plate Counts (SPC) of non-clinical goat half-udder milk samples from the studied genotypes during a complete lactation from morning and evening milking are given in tables 3 and 4, respectively. The milk of studied genotypes had medium bacterial counts. All cell counts revealed high variability especially between contagious pathogens and environmental pathogens.

The effect of genotype was significant on all contagious pathogens except Staphylococcus aureus and non significant on environmental pathogens except Bacillus measured in the morning milk samples, while, it had significant effect on environmental pathogens except for other types of Staphylococcus rather than 
agalactiae and dysgalactiae. in the evening milk samples. Damascus milk contained higher Streptococcus dysgalactiae and Streptococcus agalactiae than other genotypes (Table 3). The present study agrees with Zeng and Escobar (1995), Sung et al. (1999) and Park and Humphrey (1986), who reported that there were differences in SPC between different breeds.

Parity of doe affected significantly Corinebacteria and Streptococcus agalactiae (contagious pathogens) and did not affect all environmental pathogens in the morning milking. The influence of type of kidding was significant on most contagious and environmental pathogens (Table 3). Order of lactation month did not exhibit significant effect on different pathogens with the exception of Streptococcus dysgalactiae in both milkings and Streptococcus agalactiae in morning milking. In contrast to these results, many authors found an increase in SPC as lactation month progressed (Brown et al. (1988), Kalogridou-Vassiliadou (1991), Tirard-Collet et al. (1991), Zeng and Escobar (1995) and Zeng and Escobar (1996)).

Table 2. Evening milk samples, Least squares means, standard error ( $\pm \mathrm{SE}$ ) and probability of type I error (P) for $\log 10$ (SCC), DMY, percentages of fat, protein, lactose, total solids

\begin{tabular}{|c|c|c|c|c|c|c|c|}
\hline \multirow[t]{3}{*}{ Factor } & No & $\begin{array}{l}\log 10 \\
\text { (SCC) }\end{array}$ & DMY, kg & Fat $\%$ & Protein \% & $\begin{array}{l}\text { Lactose } \\
\%\end{array}$ & $\begin{array}{l}\text { Total } \\
\text { solid \% }\end{array}$ \\
\hline & & $\operatorname{Mean} \pm$ SE & Mean \pm SE & Mean \pm SE & Mean \pm SE & Mean \pm SE & $\operatorname{Mean} \pm$ SE \\
\hline & & $\mathbf{P}$ & $\mathbf{P}$ & $\mathbf{P}$ & $\mathbf{P}$ & $\mathbf{P}$ & $\mathbf{P}$ \\
\hline Genotype: & & 0.11 & 0.00 & 0.06 & 0.06 & 0.83 & 0.39 \\
\hline Barki(B) & 9 & $5.24 \pm .20$ & $0.229 \pm .02$ & $3.97 \pm .10$ & $3.70 \pm .09$ & $4.24 \pm .08$ & $12.12 \pm .17$ \\
\hline Damascus (D) & 37 & $5.00 \pm .15$ & $0.292 \pm .02$ & $3.77 \pm .07$ & $3.39 \pm .07$ & $4.25 \pm .06$ & $11.99 \pm .12$ \\
\hline $3 / 4 \mathrm{D} * 1 / 4 \mathrm{~B}$ & 26 & $4.89 \pm .16$ & $0.311 \pm .02$ & $3.66 \pm .08$ & $3.39 \pm .08$ & $4.28 \pm .07$ & $11.78 \pm .14$ \\
\hline $1 / 2 \mathrm{D} * 1 / 2 \mathrm{~B}$ & 15 & $5.10 \pm .22$ & $0.233 \pm .02$ & $3.60 \pm .11$ & $3.57 \pm .11$ & $4.24 \pm .09$ & $11.77 \pm .18$ \\
\hline $3 / 4 \mathrm{~B} * 1 / 4 \mathrm{D}$ & 14 & $5.52 \pm .21$ & $0.240 \pm .02$ & $3.86 \pm .11$ & $3.51 \pm .10$ & $4.35 \pm .08$ & $12.05 \pm .18$ \\
\hline Parity: & & 0.51 & 0.41 & 0.34 & 0.55 & 0.83 & 0.27 \\
\hline 1 & 26 & $5.11 \pm .18$ & $0.255 \pm .02$ & $3.73 \pm .09$ & $3.55 \pm .09$ & $4.35 \pm .07$ & $11.76 \pm .15$ \\
\hline 2 & 22 & $4.86 \pm .17$ & $0.231 \pm .02$ & $3.65 \pm .08$ & $3.53 \pm .08$ & $4.24 \pm .07$ & $11.81 \pm .15$ \\
\hline 3 & 27 & $5.13 \pm .15$ & $0.260 \pm .02$ & $3.77 \pm .07$ & $3.64 \pm .07$ & $4.28 \pm .06$ & $12.10 \pm .13$ \\
\hline 4 & 10 & $5.19 \pm .21$ & $0.286 \pm .02$ & $3.70 \pm .10$ & $3.49 \pm .10$ & $4.24 \pm .08$ & $11.81 \pm .18$ \\
\hline 5 & 4 & $5.28 \pm .35$ & $0.263 \pm .04$ & $3.87 \pm .18$ & $3.33 \pm .17$ & $4.27 \pm .14$ & $12.23 \pm .30$ \\
\hline$\geq 6$ & 12 & $5.33 \pm .21$ & $0.273 \pm .02$ & $3.92 \pm .10$ & $3.53 \pm .10$ & $4.24 \pm .08$ & $11.93 \pm .18$ \\
\hline $\begin{array}{l}\text { Type of } \\
\text { kidding: }\end{array}$ & & 0.34 & 0.57 & 0.99 & 0.76 & 0.16 & 0.62 \\
\hline 1 & 69 & $5.22 \pm .11$ & $0.257 \pm .01$ & $3.77 \pm .05$ & $3.50 \pm .05$ & $4.31 \pm .05$ & $11.97 \pm .09$ \\
\hline 2 & 32 & $5.08 \pm .14$ & $0.266 \pm .01$ & $3.77 \pm .07$ & $3.52 \pm .07$ & $4.23 \pm .06$ & $11.91 \pm .11$ \\
\hline $\begin{array}{l}\text { Month of } \\
\text { lactation: }\end{array}$ & & 0.00 & 0.00 & 0.00 & 0.00 & 0.24 & 0.01 \\
\hline 1 & 101 & $5.24 \pm .20$ & $0.199 \pm .02$ & $3.95 \pm .09$ & $3.76 \pm .10$ & $4.13 \pm .08$ & $2.04 \pm .14$ \\
\hline 2 & 87 & $5.77 \pm .18$ & $0.271 \pm .02$ & $4.15 \pm .10$ & $3.71 \pm .11$ & $4.33 \pm .06$ & $2.39 \pm .14$ \\
\hline 3 & 76 & $5.61 \pm .16$ & $0.329 \pm .02$ & $3.52 \pm .11$ & $3.27 \pm .09$ & $4.34 \pm .08$ & $11.70 \pm .15$ \\
\hline 4 & 69 & $5.14 \pm .20$ & $0.326 \pm .02$ & $3.54 \pm .10$ & $3.27 \pm .09$ & $4.35 \pm .09$ & $11.57 \pm .21$ \\
\hline 5 & 64 & $4.98 \pm .20$ & $0.286 \pm .02$ & $3.52 \pm .12$ & $3.46 \pm .08$ & $4.31 \pm .08$ & $11.63 \pm .23$ \\
\hline 6 & 51 & $4.33 \pm .26$ & $0.194 \pm .02$ & $3.77 \pm .12$ & $3.50 \pm .08$ & $4.25 \pm .09$ & $12.08 \pm .16$ \\
\hline 7 & 34 & $4.98 \pm .39$ & $0.223 \pm .02$ & $3.98 \pm .15$ & $3.61 \pm .15$ & $4.18 \pm .11$ & $12.17 \pm .17$ \\
\hline
\end{tabular}


Table 3. Morning milk samples, Least squares means, standard error ( $\pm \mathrm{SE}$ ) and probability of type I error (P) for Contagious pathogens (Corinebacteria, Streptococcus dysgalactiae, Streptococcus agalactiae and Staphylococcus aureus) and Environmental pathogens (Streptococcus uberis, Staphylococcus (other), Coliform and Bacillu.

\begin{tabular}{|c|c|c|c|c|c|c|c|c|}
\hline \multirow[t]{3}{*}{ Factor } & \multirow[t]{3}{*}{ No } & \multicolumn{7}{|c|}{ Contagious pathogens } \\
\hline & & $\begin{array}{l}\text { Corine } \\
\text { bacteria }\end{array}$ & $\begin{array}{l}\text { Strep. } \\
\text { dysgalactiae }\end{array}$ & & $\begin{array}{l}\text { Strep. } \\
\text { agalactiae }\end{array}$ & & $\begin{array}{l}\text { Staph. } \\
\text { aureus }\end{array}$ & \\
\hline & & $\begin{array}{c}\text { Mean } \pm \text { SE } \\
\mathbf{P}\end{array}$ & $\begin{array}{l}\text { Mean } \pm \text { SE } \\
\text { P }\end{array}$ & & $\underset{P}{\text { Mean }} \pm \mathrm{SE}$ & & $\begin{array}{l}\text { Mean } \pm \text { SE } \\
\quad \text { P }\end{array}$ & \\
\hline Genotype: & & 0.00 & 0.00 & & 0.00 & & 0.82 & \\
\hline Barki(B) & 9 & $5.57 \pm .06$ & $5.22 \pm .05$ & & $5.34 \pm .05$ & & $5.31 \pm .08$ & \\
\hline Damascus(D) & 37 & $5.32 \pm .06$ & $5.59 \pm .06$ & & $5.64 \pm .06$ & & $5.32 \pm .07$ & \\
\hline $3 / 4 \mathrm{D} * 1 / 4 \mathrm{~B}$ & 26 & $4.97 \pm .06$ & $5.43 \pm .05$ & & $5.45 \pm .06$ & & $5.25 \pm .08$ & \\
\hline $1 / 2 \mathrm{D} * 1 / 2 \mathrm{~B}$ & 15 & $5.33 \pm .06$ & $5.40 \pm .05$ & & $5.12 \pm .06$ & & $5.28 \pm .08$ & \\
\hline $3 / 4 \mathrm{~B} * 1 / 4 \mathrm{D}$ & 14 & $5.93 \pm .11$ & $4.97 \pm .08$ & & $5.49 \pm .07$ & & $5.48 \pm .17$ & \\
\hline Parity: & & 0.00 & 0.06 & & 0.00 & & 0.92 & \\
\hline 1 & 26 & $5.43 \pm .08$ & $5.28 \pm .06$ & & $5.28 \pm .05$ & & $5.27 \pm .10$ & \\
\hline 2 & 22 & $5.38 \pm .11$ & $5.21 \pm .15$ & & $5.51 \pm .15$ & & $5.34 \pm .14$ & \\
\hline 3 & 27 & $5.69 \pm .06$ & $5.30 \pm .05$ & & $5.12 \pm .05$ & & $5.31 \pm .08$ & \\
\hline 4 & 10 & $5.44 \pm .06$ & $5.40 \pm .05$ & & $5.57 \pm .05$ & & $5.36 \pm .08$ & \\
\hline 5 & 4 & $5.39 \pm .09$ & $5.45 \pm .07$ & & $5.52 \pm .06$ & & $5.30 \pm .12$ & \\
\hline$\geq 6$ & 12 & $5.20 \pm .06$ & $5.29 \pm .05$ & & $5.44 \pm .05$ & & $5.40 \pm .08$ & \\
\hline Type of kidding: & & 0.00 & 0.01 & & 0.00 & & 0.23 & \\
\hline 1 & 69 & $5.34 \pm .05$ & $5.38 \pm .04$ & & $5.36 \pm .05$ & & $5.29 \pm .06$ & \\
\hline 2 & 32 & $5.50 \pm .05$ & $5.27 \pm .04$ & & $5.45 \pm .05$ & & $5.37 \pm .06$ & \\
\hline $\begin{array}{l}\text { Month of } \\
\text { lactation: }\end{array}$ & & 0.44 & 0.02 & & 0.00 & & 0.50 & \\
\hline 1 & 101 & $5.31 \pm .08$ & $5.12 \pm .09$ & & $5.40 \pm .07$ & & $5.23 \pm .07$ & \\
\hline 2 & 87 & $5.49 \pm .07$ & $5.21 \pm .08$ & & $5.30 \pm .08$ & & $5.31 \pm .08$ & \\
\hline 3 & 76 & $5.38 \pm .09$ & $5.42 \pm .06$ & & $5.41 \pm .07$ & & $5.40 \pm .07$ & \\
\hline 4 & 69 & $5.49 \pm .07$ & $5.36 \pm .06$ & & $5.40 \pm .07$ & & $5.37 \pm .08$ & \\
\hline 5 & 64 & $5.43 \pm .07$ & $5.30 \pm .08$ & & $5.33 \pm .11$ & & $5.33 \pm .08$ & \\
\hline 6 & 51 & $5.38 \pm .09$ & $5.30 \pm .09$ & & $5.34 \pm .07$ & & $5.29 \pm .08$ & \\
\hline 7 & 34 & $5.48 \pm .12$ & $5.54 \pm .09$ & & $5.66 \pm .09$ & & $5.38 \pm .09$ & \\
\hline Genotype: & & & & 0.08 & & 0.12 & & 0.00 \\
\hline Barki(B) & 9 & 0.14 & $5.29 \pm .06$ & & $5.38 \pm .07$ & & $4.93 \pm .08$ & \\
\hline Damascus (D) & 37 & $5.23 \pm .07$ & $5.35 \pm .06$ & & $5.49 \pm .06$ & & $5.42 \pm .08$ & \\
\hline $3 / 4 \mathrm{D} * 1 / 4 \mathrm{~B}$ & 26 & $5.15 \pm .07$ & $5.32 \pm .06$ & & $5.45 \pm .08$ & & $5.31 \pm .09$ & \\
\hline $1 / 2 \mathrm{D} * 1 / 2 \mathrm{~B}$ & 15 & $5.42 \pm .08$ & $5.36 \pm .06$ & & $5.29 \pm .07$ & & $5.04 \pm .08$ & \\
\hline $3 / 4 \mathrm{~B} * 1 / 4 \mathrm{D}$ & 14 & $5.33 \pm .08$ & $5.75 \pm .15$ & & $5.19 \pm .15$ & & $5.26 \pm .19$ & \\
\hline & & $5.27 \pm .16$ & & & & & & \\
\hline Parity: & & & & 0.10 & & 0.59 & & 0.55 \\
\hline 1 & 26 & 0.45 & $5.57 \pm .09$ & & $5.43 \pm .09$ & & $5.18 \pm .11$ & \\
\hline 2 & 22 & $5.32 \pm .09$ & $5.37 \pm .11$ & & $5.28 \pm .13$ & & $5.24 \pm .15$ & \\
\hline 3 & 27 & $5.34 \pm .17$ & $5.47 \pm .06$ & & $5.27 \pm .07$ & & $5.23 \pm .08$ & \\
\hline 4 & 10 & $5.26 \pm .08$ & $5.46 \pm .07$ & & $5.40 \pm .08$ & & $5.28 \pm .09$ & \\
\hline 5 & 4 & $5.12 \pm .08$ & $5.34 \pm .10$ & & $5.43 \pm .11$ & & $5.16 \pm .13$ & \\
\hline$\geq 6$ & 12 & $5.31 \pm .12$ & $5.27 \pm .07$ & & $5.36 \pm .07$ & & $5.05 \pm .09$ & \\
\hline & & $5.33 \pm .08$ & & & & & & \\
\hline
\end{tabular}


Cont.

\begin{tabular}{|c|c|c|c|c|c|}
\hline \multirow[t]{3}{*}{ Factor } & \multirow[t]{3}{*}{ No } & \multicolumn{4}{|c|}{ Contagious pathogens } \\
\hline & & $\begin{array}{l}\text { Corine } \\
\text { bacteria }\end{array}$ & $\begin{array}{l}\text { Strep. } \\
\text { dysgalactiae }\end{array}$ & $\begin{array}{l}\text { Strep. } \\
\text { agalactiae }\end{array}$ & $\begin{array}{l}\text { Staph. } \\
\text { aureus }\end{array}$ \\
\hline & & $\begin{array}{c}\text { Mean } \pm \text { SE } \\
\mathbf{P}\end{array}$ & $\underset{\mathbf{P}}{\operatorname{Mean} \pm \text { SE }}$ & $\begin{array}{l}\text { Mean } \pm \text { SE } \\
\mathbf{P}\end{array}$ & $\begin{array}{l}\text { Mean } \pm \text { SE } \\
\mathbf{P}\end{array}$ \\
\hline Type of kidding: & & 0.04 & 0.07 & 0.05 & 0.33 \\
\hline 1 & 69 & $5.35 \pm .06$ & $5.47 \pm .05$ & $5.43 \pm .05$ & $5.23 \pm .06$ \\
\hline 2 & 32 & $5.20 \pm .06$ & $5.36 \pm .05$ & $5.29 \pm .06$ & $5.15 \pm .07$ \\
\hline Month of & & 0.81 & 0.76 & 0.47 & 0.99 \\
\hline lactation: & & & & & \\
\hline 1 & 101 & $5.29 \pm .08$ & $5.39 \pm .08$ & $5.33 \pm .07$ & $5.21 \pm .08$ \\
\hline 2 & 87 & $5.30 \pm .07$ & $5.41 \pm .06$ & $5.31 \pm .08$ & $5.21 \pm .09$ \\
\hline 3 & 76 & $5.38 \pm .09$ & $5.35 \pm .08$ & $5.36 \pm .06$ & $5.23 \pm .08$ \\
\hline 4 & 69 & $5.27 \pm .09$ & $5.40 \pm .05$ & $5.36 \pm .07$ & $5.18 \pm .10$ \\
\hline 5 & 64 & $5.24 \pm .09$ & $5.46 \pm .06$ & $5.36 \pm .07$ & $5.17 \pm .09$ \\
\hline 6 & 51 & $5.20 \pm .09$ & $5.43 \pm .07$ & $5.47 \pm .08$ & $5.16 \pm .10$ \\
\hline 7 & 34 & $5.27 \pm .07$ & $5.46 \pm .09$ & $5.33 \pm .11$ & $5.17 \pm .08$ \\
\hline
\end{tabular}

Table 4. evening milk samples, Least squares means, standard error ( $\pm \mathrm{SE}$ ) and probability of type I error (P) for Contagious pathogens (Corinebacteria, Streptococcus dysgalactiae, Streptococcus agalactiae and Staphylococcus aureus) and Environmental pathogens (Streptococcus uberis, Staphylococcus (other), Coliform and Bacillus)

\begin{tabular}{|c|c|c|c|c|c|c|}
\hline \multirow[t]{3}{*}{ Factor } & \multirow[t]{3}{*}{ No } & \multicolumn{5}{|c|}{ Contagious pathogens } \\
\hline & & $\begin{array}{c}\text { Corine } \\
\text { bacteria }\end{array}$ & \multicolumn{2}{|c|}{$\begin{array}{c}\text { Strep. } \\
\text { dysgalactiae }\end{array}$} & $\begin{array}{c}\text { Strep. } \\
\text { agalactiae }\end{array}$ & $\begin{array}{l}\text { Staph. } \\
\text { aureus }\end{array}$ \\
\hline & & Mean \pm SE $\quad P$ & Mean \pm SE & $\mathbf{P}$ & Mean \pm SE $\quad P$ & Mean \pm SE $\quad P$ \\
\hline Genotype: & & 0.00 & & $\overline{0.00}$ & 0.01 & 0.08 \\
\hline Barki(B) & 9 & $5.57 \pm .09$ & $5.37 \pm .03$ & & $5.48 \pm .08$ & $5.33 \pm .08$ \\
\hline Damascus (D) & 37 & $5.39 \pm .08$ & $5.37 \pm .04$ & & $5.28 \pm .07$ & $5.27 \pm .07$ \\
\hline $3 / 4 \mathrm{D} * 1 / 4 \mathrm{~B}$ & 26 & $5.03 \pm .09$ & $5.54 \pm .04$ & & $5.21 \pm .08$ & $5.17 \pm .08$ \\
\hline $1 / 2 \mathrm{D} * 1 / 2 \mathrm{~B}$ & 15 & $5.11 \pm .10$ & $5.50 \pm .04$ & & $5.10 \pm .08$ & $5.04 \pm .08$ \\
\hline $3 / 4 \mathrm{~B} * 1 / 4 \mathrm{D}$ & 14 & $5.44 \pm .13$ & $5.45 \pm .04$ & & $5.47 \pm .11$ & $5.28 \pm .11$ \\
\hline Parity: & & 0.93 & & 0.00 & 0.82 & 0.51 \\
\hline 1 & 26 & $5.40 \pm .11$ & $5.59 \pm .04$ & & $5.39 \pm .10$ & $5.30 \pm .10$ \\
\hline 2 & 22 & $5.26 \pm .16$ & $5.46 \pm .11$ & & $5.27 \pm .12$ & $5.27 \pm .13$ \\
\hline 3 & 27 & $5.32 \pm .08$ & $5.28 \pm .03$ & & $5.29 \pm .07$ & $5.21 \pm .07$ \\
\hline 4 & 10 & $5.25 \pm .09$ & $5.51 \pm .03$ & & $5.33 \pm .08$ & $5.27 \pm .08$ \\
\hline 5 & 4 & $5.32 \pm .15$ & $5.51 \pm .04$ & & $5.35 \pm .12$ & $5.04 \pm .11$ \\
\hline$\geq 6$ & 12 & $5.31 \pm .08$ & $5.31 \pm .04$ & & $5.22 \pm .07$ & $5.22 \pm .07$ \\
\hline $\begin{array}{l}\text { Type of } \\
\text { kidding: }\end{array}$ & & 0.64 & & 0.00 & 0.91 & 0.67 \\
\hline 1 & 69 & $5.29 \pm .06$ & $5.50 \pm .03$ & & $5.30 \pm .05$ & $5.23 \pm .05$ \\
\hline 2 & 32 & $5.33 \pm .07$ & $5.39 \pm .03$ & & $5.31 \pm .06$ & $5.20 \pm .06$ \\
\hline $\begin{array}{l}\text { Month of } \\
\text { lactation: }\end{array}$ & & 0.20 & & 0.01 & 0.73 & 0.68 \\
\hline 1 & 101 & $5.27 \pm .07$ & $5.34 \pm .06$ & & $5.28 \pm .06$ & $5.14 \pm .07$ \\
\hline 2 & 87 & $5.18 \pm .07$ & $5.42 \pm .06$ & & $5.37 \pm .06$ & $5.22 \pm .07$ \\
\hline 3 & 76 & $5.29 \pm .07$ & $5.45 \pm .05$ & & $5.37 \pm .07$ & $5.31 \pm .07$ \\
\hline 4 & 69 & $5.28 \pm .08$ & $5.51 \pm .04$ & & $5.31 \pm .06$ & $5.19 \pm .07$ \\
\hline 5 & 64 & $5.42 \pm .09$ & $5.45 \pm .07$ & & $5.26 \pm .07$ & $5.26 \pm .08$ \\
\hline 6 & 51 & $5.38 \pm .10$ & $5.32 \pm .07$ & & $5.24 \pm .07$ & $5.22 \pm .08$ \\
\hline 7 & 34 & $5.34 \pm .09$ & $5.63 \pm .07$ & & $5.32 \pm .08$ & $5.19 \pm .12$ \\
\hline
\end{tabular}


Cont.

\begin{tabular}{|c|c|c|c|c|c|}
\hline \multirow[t]{3}{*}{ Factor } & \multirow[t]{3}{*}{ No } & \multicolumn{4}{|c|}{ Contagious pathogens } \\
\hline & & $\begin{array}{c}\text { Corine } \\
\text { bacteria }\end{array}$ & $\begin{array}{c}\text { Strep. } \\
\text { dysgalactiae }\end{array}$ & $\begin{array}{c}\text { Strep. } \\
\text { agalactiae }\end{array}$ & $\begin{array}{l}\text { Staph. } \\
\text { aureus }\end{array}$ \\
\hline & & Mean \pm SE $\quad P$ & Mean \pm SE $\quad P$ & Mean \pm SE $\quad P$ & Mean \pm SE $\quad P$ \\
\hline \multicolumn{6}{|l|}{ Genotype: } \\
\hline Barki(B) & 9 & 0.00 & 0.76 & 0.00 & 0.03 \\
\hline Damascus & 37 & $5.32 \pm .04$ & $5.35 \pm .06$ & $5.00 \pm .08$ & $5.19 \pm .06$ \\
\hline (D) & 26 & $5.64 \pm .04$ & $5.39 \pm .06$ & $5.42 \pm .07$ & $5.14 \pm .07$ \\
\hline $3 / 4 \mathrm{D} * 1 / 4 \mathrm{~B}$ & 15 & $5.43 \pm .04$ & $5.36 \pm .07$ & $5.34 \pm .09$ & $5.39 \pm .07$ \\
\hline $1 / 2 \mathrm{D} * 1 / 2 \mathrm{~B}$ & 14 & $5.43 \pm .05$ & $5.44 \pm .07$ & $4.89 \pm .08$ & $5.38 \pm .08$ \\
\hline $3 / 4 \mathrm{~B} * 1 / 4 \mathrm{D}$ & & $4.79 \pm .05$ & $5.44 \pm .09$ & $5.03 \pm .12$ & $5.16 \pm .10$ \\
\hline \multicolumn{6}{|l|}{ Parity: } \\
\hline 1 & 26 & 0.00 & 0.08 & 0.96 & 0.00 \\
\hline 2 & 22 & $5.25 \pm .04$ & $5.58 \pm .08$ & $5.16 \pm .11$ & $5.00 \pm .08$ \\
\hline 3 & 27 & $5.27 \pm .14$ & $5.35 \pm .11$ & $5.15 \pm .13$ & $5.39 \pm .16$ \\
\hline 4 & 10 & $5.31 \pm .04$ & $5.38 \pm .06$ & $5.07 \pm .07$ & $5.23 \pm .06$ \\
\hline 5 & 4 & $5.42 \pm .04$ & $5.34 \pm .06$ & $5.14 \pm .08$ & $5.19 \pm .06$ \\
\hline \multirow[t]{2}{*}{$\geq 6$} & 12 & $5.39 \pm .05$ & $5.45 \pm .10$ & $5.18 \pm .13$ & $5.30 \pm .10$ \\
\hline & & $5.29 \pm .04$ & $5.25 \pm .06$ & $5.11 \pm .08$ & $5.41 \pm .06$ \\
\hline \multicolumn{6}{|l|}{ Type of } \\
\hline $\begin{array}{r}\text { KIddıng: } \\
1\end{array}$ & 69 & $\begin{array}{c}0.00 \\
5.39 \pm .04\end{array}$ & $\begin{array}{c}0.01 \\
5.47 \pm .04\end{array}$ & $\begin{array}{c}0.36 \\
5.17 \pm .05\end{array}$ & $\begin{array}{c}0.00 \\
5.34 \pm .05\end{array}$ \\
\hline 2 & 32 & $5.26 \pm .04$ & $5.32 \pm .05$ & $5.10 \pm .06$ & $5.17 \pm .05$ \\
\hline \multicolumn{6}{|l|}{ Month of } \\
\hline lactation: & & 0.30 & 0.19 & 0.74 & 0.70 \\
\hline 1 & 101 & $5.21 \pm .07$ & $5.34 \pm .06$ & $5.11 \pm .07$ & $5.26 \pm .07$ \\
\hline 2 & 87 & $5.27 \pm .07$ & $5.38 \pm .06$ & $5.19 \pm .06$ & $5.30 \pm .06$ \\
\hline 3 & 76 & $5.34 \pm .06$ & $5.29 \pm .07$ & $5.20 \pm .08$ & $5.33 \pm .08$ \\
\hline 4 & 69 & $5.37 \pm .06$ & $5.39 \pm .05$ & $5.17 \pm .07$ & $5.19 \pm .09$ \\
\hline 5 & 64 & $5.33 \pm .07$ & $5.43 \pm .06$ & $5.14 \pm .08$ & $5.23 \pm .07$ \\
\hline 6 & 51 & $5.27 \pm .08$ & $5.48 \pm .08$ & $5.06 \pm .09$ & $5.26 \pm .07$ \\
\hline 7 & 34 & $5.47 \pm .07$ & $5.44 \pm .09$ & $5.08 \pm .08$ & $5.20 \pm .07$ \\
\hline
\end{tabular}

\section{Correlation coefficients}

Partial correlation coefficients between all studied traits are presented in tables 5 and 6 for morning and evening milkings, respectively. The present results agree with that of Zeng et al. (1997), who reported that DMY had a negative relationship with SCC, while SCC had a positive relationship with fat \%, protein \% and total solids \%. Sung et al. (1999) reported that the correlation coefficients between SCC and milk composition were highly significant $(p<0.001)$ for Alpine, Nubian, Saanen and Toggenburg in Taiwan. Tables 5 and 6 indicated that SCC did not strongly correlated with SPC, may be due to low number of samples and therefore, the correlations obtained in this study are considered as preliminary results. Park and Humphrey (1986) found none significant correlation coefficients between SCC and bacteria cell 
counts for the pooled data from Alpine and Nubian does. They showed that the correlation coefficients between SCC and fat $\%$ and protein $\%$ were significant $(p<0.01)$ for combined or separated breed data. In contrast to the results in this study, Zeng and Escobar (1995) reported that SCC had a positive relationship $(p<0.001)$ with SPC and a negative relationship $(p<0.001)$ with fat $\%$, protein $\%$, total solids $\%$, lactose \% and milk production. Zeng and Escobar (1996) reported that SCC of Alpine goat positively correlated with SPC, while it did not correlate with SPC in Nubian milk. SPC had no correlation with any of the milk components of the combined breed data.

\section{CONCLUSION}

The analyzed data set did not find significant effect of genotype on milk yield traits including SCC with the exception of DMY in the evening milking, while most of the contagious and environmental bacteria were affected by the genotype effect. Order of lactation month was among the most important factors contributing to SCC, DMY and milk composition variation although the means through months of lactation did not show any particular trend.

\section{ACKNOWLEDGEMENT}

The authors are grateful to Prof. Dr. E.S.E. Galal for his helpful and advice in statistical analysis and criticism of the manuscript of the work.

\section{REFERENCES}

Abo-Ismail, A., 2003. Genetic and environmental factors affecting yield and odor of milk and selection indices in goats. M.Sc. Thesis. Fac. Aric., Alex. Univ., Egypt.

Ali, A.K.A., and G.E. Shook, 1980. An optimum transformation for somatic concentration in milk. J. Dairy Sci. 63: 487-490.

Brown, D.L., S.K. Lomison. and G.E. Bradford, 1988. Effect of ambient temperature on milk production of Nubian and Alpine goats. J. Dairy Sci. 71: 2486-2490.

Droke, E.A., M.J. Paape and Di Carlo, 1993. Prevalence of high somatic cell counts in bulk tank goat milk. J. Dairy Sci. 76: 1035-1039.

Dulin, A.M., M.J. Paapa, W.D. Schultz and B.T. Weinland, 1983. Effect of parity, stage of lactation, and intramammary infection on concentration of somatic cells in goat milk. J. Food Prot. 45: 435-439.

El-Saied, U.M., R.E. Al-Ahwal, M.E. Ahmed, Ferial Hassan and B.M. Edris, 2003. Relationship between somatic cell count and intramammary infection in Egyptian Zaraibi goats. Egyptian J. Anim. Prod. 40: 129-137.

Haenlein, G.F.W., 2000. Role of goat meat and milk in human nutrition. Proceedings $\mathrm{V}^{\text {th }}$ International Conference on Goats, New Delhi, India, March 1-8, ICAR Publ., New Delhi, 2 (II): 575-580.

Haenlein, G.F.W., and L.S. Hinkely, 1995. Goat milk somatic cell count situation in USA. Proc. Conf. on somatic cell count and milk of Small Ruminants, Bella, Italy, 1994. p17. 
Hatfield, P.G., G.D. Snowder, W.A. Head, H.A. Glimp, R.H. Stobart and T. Besser, 1995. Production of ewes rearing twin lambs: Effect of dietary crude protein percentage and supplemental zinc methionine. J. Anim. Sci. 73: 1227-1238.

Hayden, T.J., C.R. Thomas and I.A. Forsyth, 1979. Effect of number of young born (litter size) on milk of goats: role of placental lactogen. J. Dairy Sci. 62: 53-57.

Higgs, T.M. and A.J. Bramley, 1981. Laboratory techniques for examination of milk samples. In : Mastitis control and Herd Management. Technical Bulletin 4, NIDR, Reading, UK. PP 94-109.

Houghtby, G.A., I.J. Martin and E.K. Koenig, 1992. Microbiological count methods. In. R.T. Marshall (Editor), Standard Methods for the Examination of Dairy Products. $16^{\text {th }}$ edn. American Public Health Association (APHA). Washington.DC. PP.243 -246.

Kalogridou-Vassiliadou, D., 1991. Mastitis-related pathogens in goat milk. Small Ruminant Res. 4: 203-212.

Maisi, P., 1990. Milk NAGase, CMT and antitrypsin as indicators of caprine subclinical mastitis infection. Small Ruminant Res. 3: 493-501.

Osman, Mona. A., U.M. EI-Saied, M. EIsayed and A.A. Raheem, 2004. Comparison of Zaraibi, Damascus and Baladi goats in milk composition and somatic cell counts Egyptian J. Anim. Prod. 41: 39-48.

Park, Y.W. and R.D. Humphrey, 1986. Bacterial Cell Count in goat milk and their correlations with somatic cell counts, percent fat and protein. J. Dairy Sci. 69: 3237.

PMO, 1993. Grade A Pasteurized Milk Ordinance. US Department of Health and Human Services, Washington, DC.

Poutrel, B., R. de Cremoux, M. Ducelliez and D. Verneau, 1997. Control of intramammary infections in goats: Impact on somatic cell counts. J. Anim. Sci. 75: 566-570.

Prasad, H. and O.P.S. Sengar, 2002. Milk yield and composition of the Barbari goat breed and its crosses with Jamunapari, Beetal and Black Bengal. Small Ruminant Res. 45: 79-83.

SAS, 1996. SAS User Guide, Statistics. Version 8 edition SAS Institute INC. Cary NC.

Sheldrake, R.F., R.J.T. Hoare and V.E. Woodhouse, 1981. Relation of somatic cell count and cell volume analysis of goat's milk to intramammary infection with coagulase-negtive staphylococci. J. Dairy. Sci. 48: 393-403.

Sung, Y.Y., T.I. Wu and P.H. Wang, 1999. Evaluation of milk quality of Alpine, Nubian, Saanen and Toggenburg breeds in Taiwan. Small Ruminant Res. 33: 1723.

Tirard-Collet, P., J.A. Zee, L. Carmichael and R.E. Simard, 1991. A study of the microbiological quality of goat milk in quebec. J. Food. Prot. 54: 263-266.

Wilson, D.J., K.N. Stewart and P.M. Sears, 1995. Effects of stage of lactation, production, parity and season on somatic cell counts in infected and uninfected dairy goats. Small Ruminant Res. 16: 165-169.

Zeng, S.S. and E.N. Escobar, 1995. Effect of parity and milk production on somatic cell count, standard plate count and composition of goat milk. Small Ruminant Res. 17: 269-274. 
Zeng, S.S. and E.N. Escobar, 1996 Effect of breed and milking method on somatic cell count, standard plate count and composition of goat milk. Small Ruminant Res. 19: 169-175.

Zeng, S.S., E.N. Escobar and T. Popham, 1997. Daily variations in somatic cell count, composition and production of Alpine goat milk. Small Ruminant Res. 26: 253-260. 


\title{
تأثير التركيب الوراثى ويعض العوامل البيئية على محصول اللبن وتركيب اللبن وتعداد

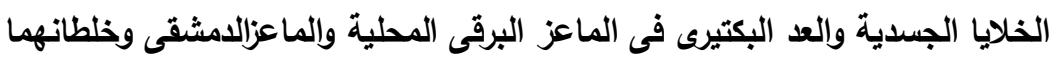

\author{
محمد محمد عيسى ، منى عبد الظاهر عثمان

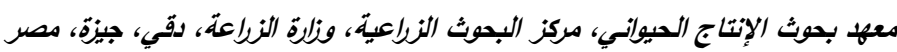

دُرست الماعز البرقى الصحراوى والدمشقى والجيل الأول (خلبط 1/2 دمشقى * 1/2 برقى) والخلطان الرجعية

(3/4 دمشقى *1/4 برقى)، (3/4 برقى *1/4 دمشقى) تحت الظروف شبه الجافة للمنطقة الساحلية بالصحراء الغربية

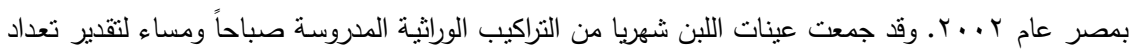
الخلايا الجسدية ومحصول اللبن اليومى والنسب الئوية للدهن والبروتين واللاكتوز والمواد الصلبة الكلية وتعداد الخلايا البكتيرية. وقدر أيضا معامل الارتباط الجزئى بين جميع الصفات المدروسة. أثنارت متوسطات مجموع المربعات الصغرى الى أن الماعز الدمشقى أنتج محصول لبن يومى أعلى من البرقى وذللك فى حلبة الصباح

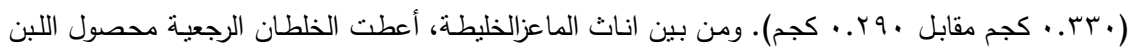
اليومى الأعلى مقارنة بخليط 1/2 دمشقى * 1/2 برقى. وكان تأثثر التركيب الوراثى معنويا على البروتين واللاكتوز

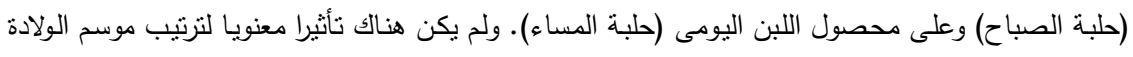

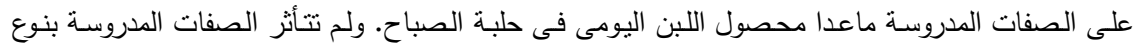
الولادة سوى اللاكتوز فى عينات لبن الصباح. وقد كان لترتيب شهور الحليب تأثثرا معنويا على تعداد الخلايا

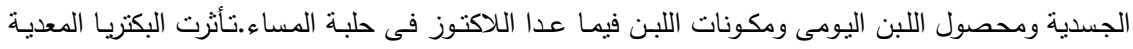
بالتركيب الوراثى بإسنثناء Staph. aureus. وكان لترتيب موسم الولادة تأثنراً معنوياً على Corinebacteria ذوع في حلبة الصباح وعلى Strep. dysgalactiae فى حلبة المساء. وكان تأثير

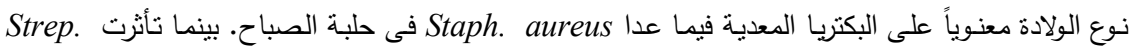

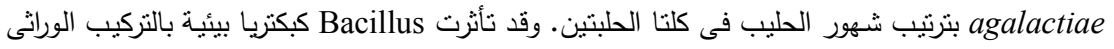
فى الحلبتين بينما نأثرت Strep. uberis بنوع الولادة. ولم يظهرلترتيب شهور الحليب نتأثيراً معنوياً على البكتريا البيئية. وقد كان لتعداد الخلايا الجسدية علاقة موجبة مع كل من نسبة الدهن والبروتين والمواد الصلبة الكلية والبكتريا المعدية، بينما كان لها علاقة سالبة مع محصول اللبن اليومى واللاكتوز وذللك فى الحلبتين. وارتبط محصول اللبن اليومى سلبيا مع نسبة الدهن والبروتين. ويعتبر تعداد الخلايا الجسدية من أهم الطرق لتقيم الحالة الصحية للضرع. 
Egyptian J. Anim. Prod., 43, Suppl. Issue, Dec. (2006):65-79

Issued by The Egyptian Society of Animal Production 